\title{
Knee Preservation and Restoration Versus Replacement
}

\section{Prakash*}

Director and Chief of Orthopaedics, Institute for Special Orthopaedics, India

*Corresponding Author: L Prakash, Director and Chief of Orthopaedics, Institute for Special Orthopaedics, India.

Received: September 17, 2019; Published: October 01, 2019

DOI: 10.31080/ASOR.2019.02.0108

Total knee replacement is the fastest growing "High Cost" orthopedic surgery in the world [1]. In India, the per annum knee replacements have multiplied thirty times in the last decade [2]. With such large numbers of knee replacements being performed regularly, many of them on patients younger than 55 years, it is logical that in years to come, there will arise a need for a large number of revision surgeries, which are far more complex and costlier than the index primary surgery [3].

With total knee replacement TKR surgery being covered by all insurance schemes, including the government schemes [4], younger surgeons are in a rat race to perform this surgery even on those patients on whom it is not indicated. Camps are being held and public awareness campaigns are being run by orthopedic surgeons and hospitals extolling the benefits of this procedure, and warning the patients of the perils of its delay (Figure 1).

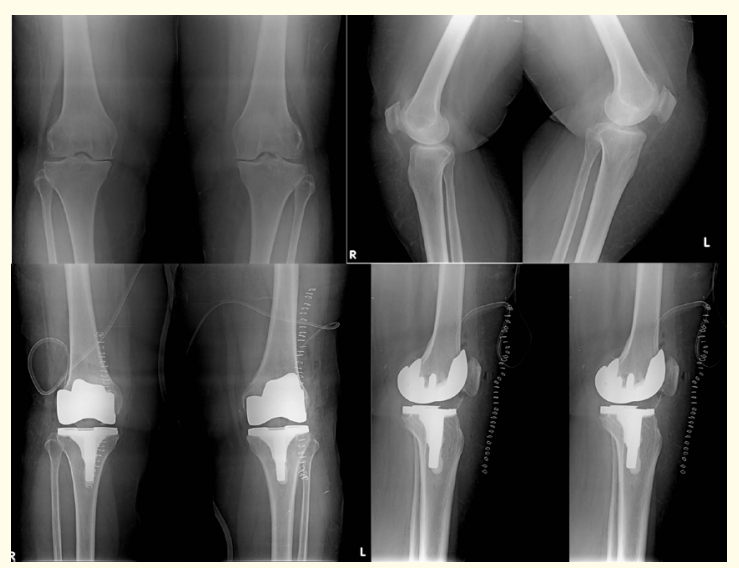

Figure 1: Knee replacement is an aggressively promoted surgery.

However the risks, complications, and the natural history of the artificial knee joints are seldom discussed in detail with the patients. Likewise few surgeons inform the younger patients about the finite life of the artificial knee, and the extremely high cost, technical skills and infrastructure needed for revision knee replacements.
This results in a situation where near normal knees are replaced for minimal symptoms without any attempts at restoration or preservation of the joint. Despite the fact that logic tells us that a natural knee is always preferable to an artificial one, the number of surgeons engaged in preservation and restoration are far fewer than the primary arthroplasty surgeon. Arthroplasty today is a glamorous sub specialty and most young trainees aspire to be a replacement surgeon, rather than a restoration/preservation surgeon.

The X-ray in figure 2 shows a pre and post operative X-rays of both knees of a doctors mother which underwent replacement. Though the post operative radiograph has nothing to criticize on technical grounds, a look at the preoperative X-ray will clearly indicate that the patient is suffering from only very early minimal medial compartment OA of the knee. Such knees should not be replaced.

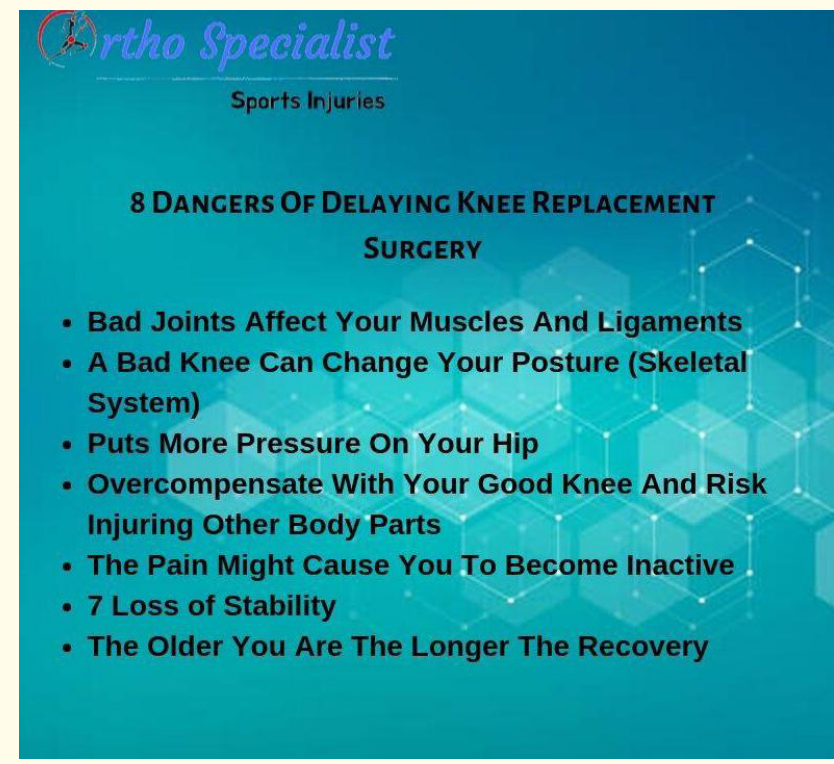

Figure 2: A knee replacement has been performed on a near normal knee which could have been managed better by other lesser invasive methods. 
Traditional wisdom by pioneers of knee arthroplasty has always taught us that TKR should be reserved as a last resort in management of knee OA, after exhausting all other methods including minimally invasive or non destructive surgeries [5]. The following methods of knee preservation have been shown to improve the clinical symptoms and either postpone or avoid knee replacement surgery.

1. Medical management by Mukherjee regimen

2. Stem cell cartilage regeneration

3. Proximal fibular Osteotomy

4. High Tibial Osteotomy.

Varus knees far outnumber valgus knees, making medial compartment osteoarthritis of the knee far commoner than all other types. The methods mentioned above selectively target the diseased part of the joint, without interfering with the new normal lateral compartment and the patello-femoral joint.

Medical management by reducing the rapidly proliferating inflammatory cells and augmenting the bone quality by Mukharjee regimen [6] has shown promising results and is still being evaluated. Likewise stem cell therapy is an established procedure which is being regularly performed in a few select centers with promising early short term results [7]. However both these procedures need a longer follow up and larger numbers to prove their efficiency.

PFO or proximal fibular osteotomy (Figure 3) is a very simple procedure that aims to remove the lateral deforming forces by resecting a small part of fibula. This is an excellent procedure which has shown to produce outstanding short term results in the form of pain relief and function restoration [8-12]. However the indications are very specific and the band width of patients falling into the category of those who will benefit from this procedure is rather narrow. Improper case selection is doomed to failure, bringing an ill reputation to this otherwise excellent procedure [12]. One more troubling aspect of this surgery is transient neuropraxia or weakness of EHL, which lasts from few weeks to few months. This can be avoided by very careful surgical technique and meticulous adherence to correct steps [12].

HTO or high tibial osteotomy is the gold standard for medial compartment OA with Varus, and is known to correct the deformity and produce excellent relief from symptoms and restoration of function [13]. The osteotomy can be fixed by internal plates or external apparatus and bot have shown to produce remarkable results lasting 15 years or longer [12-14]. This excellent procedure has gradually fallen out of favour because it is technically more demanding than the very simple steps fo a total knee replacement.

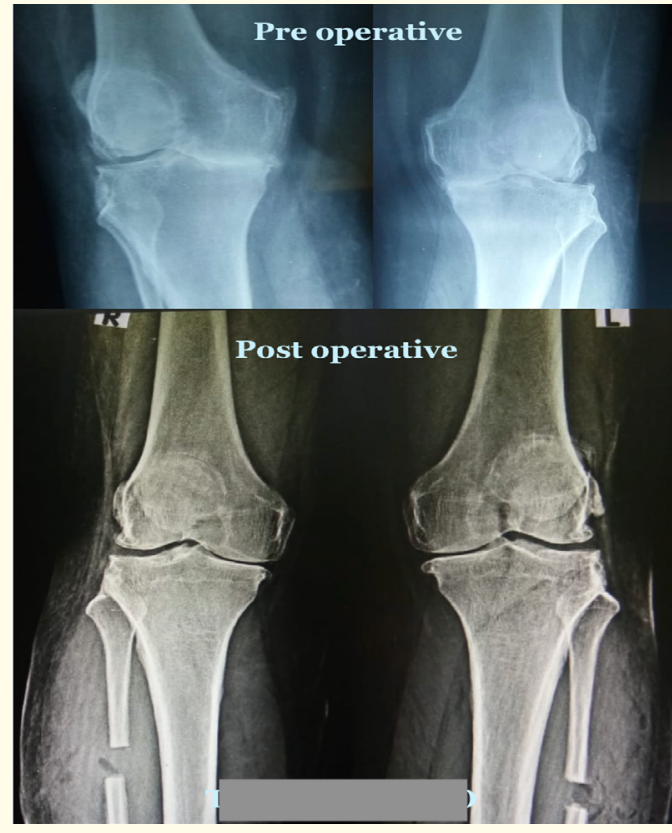

Figure 3: PFO or proximal fibula osteotomy restores medial joint space and produces dramatic pain relief in correctly selected cases.

In conclusion, the younger surgeons should invest in learning methods of joint preservation and restoration, rather than being enamored with the glamours of arthroplasty, and offer these as the first line of management to all cases of early OA knees. Total Knee Arthroplasty should be reserved for gross tricompartmental arthritis in the elderly to avoid the overwhelming burden of revision surgeries in the near future [5].

\section{Bibliography}

1. Maradit Kremers H., et al. "Prevalence of Total Hip and Knee Replacement in the United States". The Journal of bone and joint surgery American 97.17 (2015): 1386-1397.

2. Inacio MCS., et al. "Projected increase in total knee arthroplasty in the United States - An alternative projection model". Osteoarthritis and Cartilage 25.11 (2017): 1797-1803.

3. Prakash L. "A beginner's guide to total knee replacement". CBS Publications, New Delhi (2016).

4. Cram P., et al. "Total knee arthroplasty volume, utilization, and outcomes among Medicare beneficiaries, 1991-2010". Journal of the American Medical Association 308.12 (2012): 12271236. 
5. Cordtz RL., et al. "Incidence of hip and knee replacement in patients with rheumatoid arthritis following the introduction of biological DMARDs: an interrupted time-series analysis using nationwide Danish healthcare registers". Annals of the Rheumatic Diseases 77.5 (2018): 684-689.

6. Mukharjee A. "Non surgical management and reversal of AVN by Mukharjee Regimen". Chapter from book. Indian Academy of Orthopaedic Surgeons Chennai India (2019).

7. Zhang Y., et al. The pathogenesis research of non-uniform settlement of the tibial plateau in knee degeneration and varu (2018).

8. Prakash L. "Proximal Fibular Osteotomy". Indian Academy of Orthopaedic Surgeons, Instructional course lectures (2016).

9. Yang ZY., et al. "Medial Compartment Decompression by Fibular Osteotomy to Treat Medial Compartment Knee Osteoarthritis: A A Pilot Study". Orthopaedics 38.12 (2015): e1110e1114.

10. Hofmann S., et al. "Osteotomies of the knee joint in patients with monocompartmental arthritis". Orthopaedic 38.8 (2009): 755-769.

11. Wei XC., et al. "Proximal Fibular Osteotomy, A New Surgery for Pain Relief and Improvement of Joint Function in Human Knee Osteoarthritis". Journal of International Medical Research 45.1 (2017): 282-289.

12. Prakash L. "Orthopaedics Usual and unusual". Indian Academy of Orthopaedic Surgeons, Instructional course lectures (2016).

13. Wu LD., et al. "A long- term follow-up study of high tibial osteotomy for medial compartment osteoarthrosis". Chinese Journal of Traumatology 7.6 (2004): 348-353.

14. Sprenger TR and Doerzbacher JF. "Tibial oste- otomy for the treatment of varus gonarthro- sis: survival and failure analysis to twenty- two years". Journal of Bone and Joint Surgery American 85.3 (2003): 469-474.

Volume 2 Issue 11 November 2019

(C) All rights are reserved by L Prakash. 